\title{
Classification of Voltage Sag Using Multi-resolution Analysis and Support Vector Machine
}

\author{
Hanim Ismail, Zuhaina Zakaria, and Noraliza Hamzah
}

\begin{abstract}
Voltage sag disturbance can cause catastrophe failures to both utility and end users of electrical power. The causes of this disturbance include power system fault condition and induction motor starting. This paper presents a research to classify the causes of voltage sag by employing Multi Resolution Analysis (MRA) and Support Vector Machines (SVM). Voltage sag data are obtained from the implementation of various fault conditions and induction motor starting using PSCAD modeling and data generation. Multi Resolution Analysis (MRA) is developed as a representation of a signal with various levels of decomposition for the features extraction. The two features used are the minimum and maximum of voltage and the energy distribution of ten decomposition levels of the MRA. Those features are used as the input for classification using Support Vector Machines (SVM). In classification part, the Radial Basis Function (RBF) kernel of the SVM has been used. The result shows that the MRA and SVM can classify the source of voltage sag with promising accuracy.
\end{abstract}

Index Terms-Power quality, voltage sag, multi resolution analysis, support vector machine.

\section{INTRODUCTION}

Voltage sag disturbance is one of the most frequent power quality problems which occur between a few tens and several hundred times per year [1]. Voltage sags are typically caused by fault conditions [2], in which short-circuit faults and earth faults are found to cause severe voltage sags [3]. In industrial and commercial power systems, faults on one-feeder tend to cause voltage drops on all other feeders in the plant [4]. Identifying the root of voltage sag problem has been in the fore front research area in power system. Support Vector Machine (SVM) has been used in power quality disturbance classification [5]-[8]. Reference [6] proposed a SVM classification for voltage disturbance. Data from voltage disturbances for faults and transformer energizing are used and the triggering point of disturbance, frequency magnitude and the total harmonic distortion (THD) are used as the input for the SVM. The faults in each case have been grouped and testing has been carried out separately to verify the performance of this method [6]. General classification of power quality disturbance has been proposed in [6]-[8] in which power quality disturbance such as swell, flicker, harmonics and voltage sag were classified. The studies which narrow down to classify the cause of voltage sags have been presented in [9]-[11]. Reference [9] presents a new classification of voltage sags mathematically justified by

Manuscript received March 31, 2015; revised July 17, 2015.

H. Ismail, Z. Zakaria, and N. Hamzah are with Universiti Teknologi MARA, Malaysia (e-mail: hanim_ismail@yahoo.com, noralizah@salam.uitm.edu.my, zuhainaz@salam.uitm.edu.my). introducing two new indices, namely, phase-to-neutral and phase-to-phase voltage indices. The proposed classification is based on characteristic voltage, zero-sequence components of voltage and type of voltage sag. Implementation of the method needs details characteristics and the performance in terms of accuracy percentage has not been presented [9]. Another proposed method to classify the voltage sag based on signal processing approach is presented in reference [10] whereby generalized S-transform windowing technique has been implemented to determine the cause. Such method needs sample of the frequency spectrum of the S-transform and matching procedure to classify the cause of the sag cannot be automatically done. References [11], [12] present an algorithm to detect and classify the causes of voltage sag using on Empirical Mode Decomposition (EMD) and Probability Neural Network. Similarly with [6], the voltage sag data has been grouped and results are based on group identification. The results in [11] are promising but the voltage sags data are divided into particular groups. This paper presents the classifying of the causes of voltage sag using Multi Resolution Analysis (MRA) and Support Vector Machines (SVM). The wavelet transformation will be utilized as feature extraction based on the MRA coefficient and the SVM will be used to classify the cause of the voltage sag. This method use randomly selected voltage sag data and is not grouped as in [6], [11]. Synthetic data based on two established standard IEEE test systems i.e., the IEEE 30 bus systems and IEEE 34 node distribution system are used to justify the method.

\section{MULTI-RESOLUTION ANALYSIS}

In this research MRA is used to develop the representations of the voltage sag signal at various levels of resolutions. The signal will be filtered at each level by employing low pass filter (LPF) and high pass filter (HPF). The signal is denoted by the $C_{0}[n]$, where $\mathrm{n}$ is an integer is distributed in three levels. The high pass filter is denoted by $G_{0}$ while the low pass filter is denoted by $H_{0}$ as in Fig. 1 .

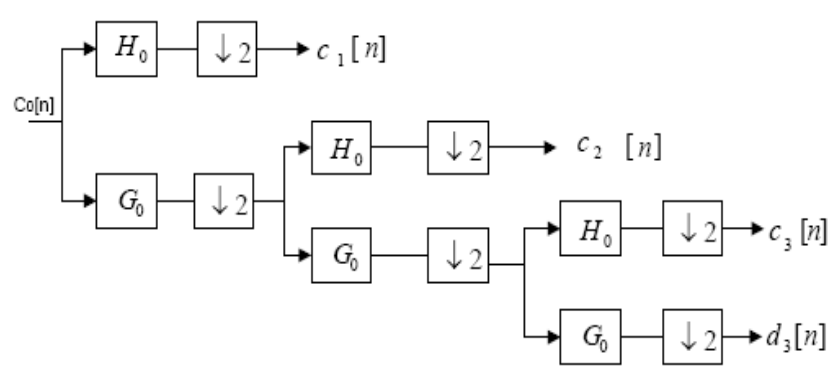

Fig. 1. Low-pass and high-pass filter of the discrete time signal. 
Upon filtering, the signal is decomposed starting from level 1 onwards. The decomposition coefficients of MRA analysis which correspond to the decomposition of signal $x(t)$ is expressed as,

$$
\begin{gathered}
x(t)=A_{1}(t)+D_{1}(t) \\
=A_{2}(t)+D_{2}(t)+D_{1}(t) \\
=A_{3}(t)+D_{3}(t)+D_{2}(t)+D_{1}(t)
\end{gathered}
$$

In this paper, the voltage sag signals were transformed into six different resolution levels and the decomposition detail level $\mathrm{d} 1$ has been chosen as it gives the best accurate beginning time for voltage sag. Fig. 2 shows the original waveform is decomposed into approximate a and detail coefficients from $\mathrm{d} 1$ to $\mathrm{d} 6$. The first type of features extracted from the detail level $1(\mathrm{~d} 1)$ is the minimum and the maximum value of voltage. The second features is the energy extracted from those levels.

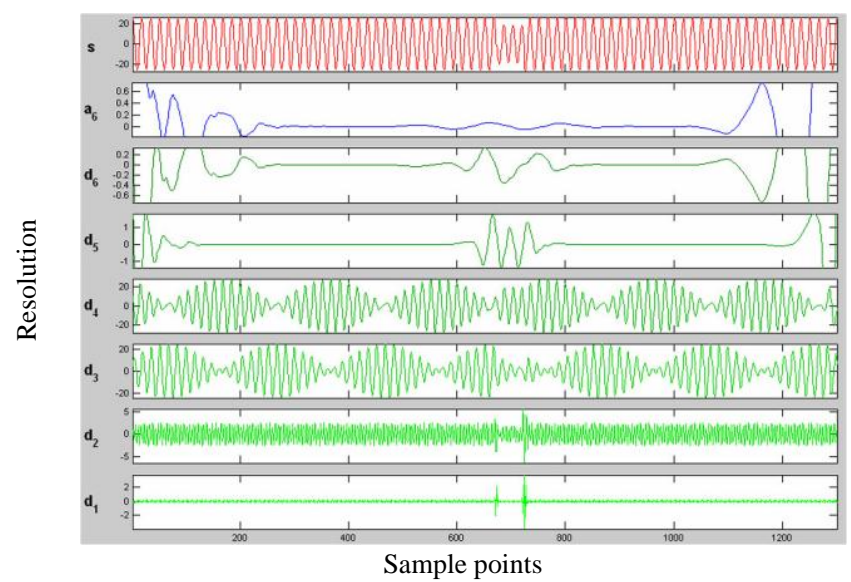

Fig. 2. Voltage sag and wavelet transformation at six resolution levels.

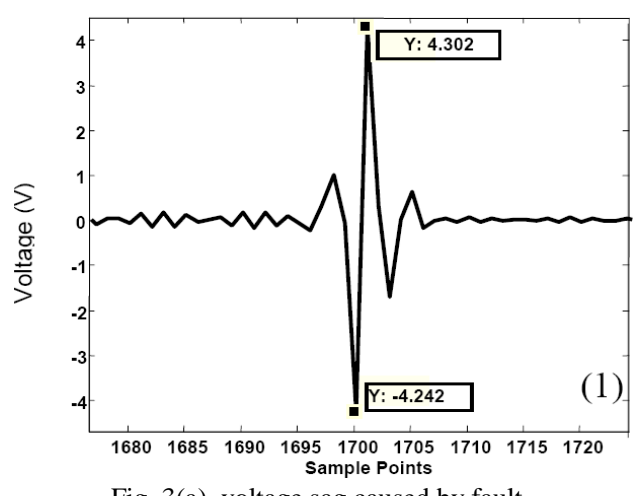

Fig. 3(a). voltage sag caused by fault.

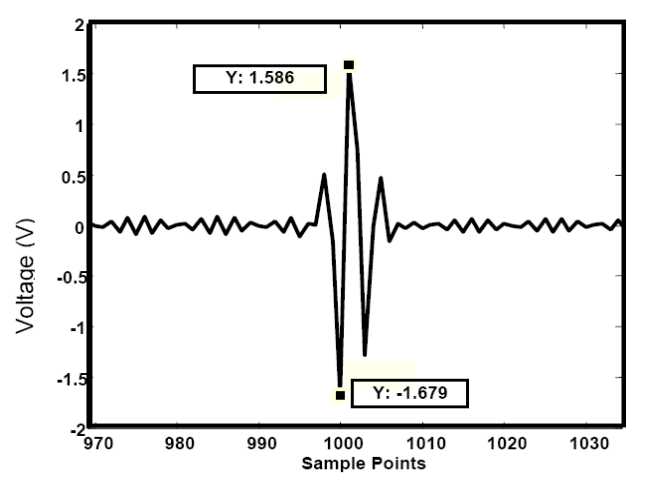

Fig. 3(b). Voltage sag caused by starting of induction motor.
Fig. 3(a) and Fig. 3(b) shows the feature extraction of the minimum and maximum point of the starting of sag caused by the fault and induction motor starting respectively obtained from IEEE 30 bus system. Fig. 4(a) and Fig. 4(b) shows the energy distribution for fault and induction motor starting respectively. From both figures, significant energy levels are shown occurring at level 3 and 4.

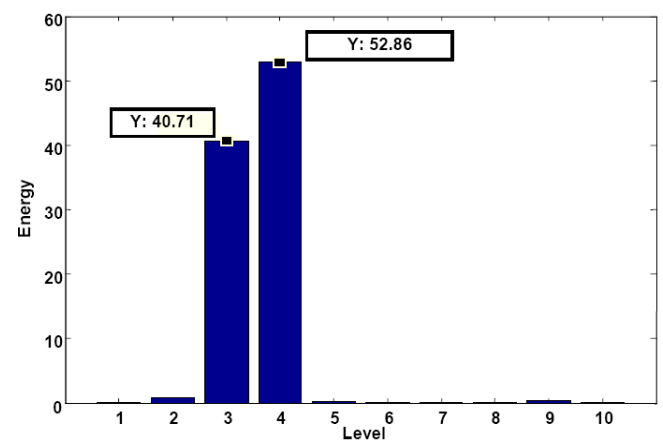

Fig. 4(a). Energy of voltage sag caused by fault.

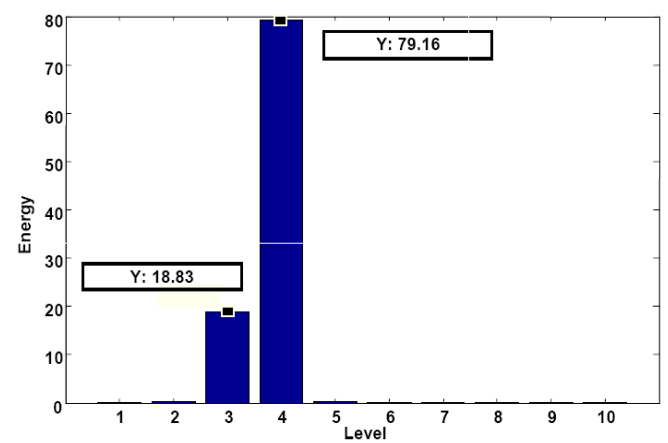

Fig. 4(b). Energy of voltage sag caused by induction motor

\section{ClassificATION USING SVM}

Support Vector Machines (SVM) are supervised learning algorithms pioneered by Vapnik [13]. The principle of SVM is to find a decision boundary for separating two classes of voltage sag and minimize the classification error on the selected features. A classification task involves separating data into training and testing sets. Each instance in the training set contains one class label (target value) and several attributes (features). The main goal of SVM is to produce a model (based on the training data) which will then is used to predict the class label of the test data. Both training and testing set are first scaled and normalized to avoid numerical difficulties. Next, the training set is trained to find the best parameter and become a model of the classifier. Finally, the testing set is used to predict the class label of the test data.

Given the training data,

$$
\left(x_{1}, y_{1}\right), \ldots,\left(x_{l}, y_{l}\right), x \in R^{M}
$$

where each data consists of $M$ features. These features describes for a two-class problems, as

$$
y_{i} \in\{-1,+1\}
$$

The SVM constructs the decision function given as $g(x)=\langle w \times x\rangle+b$, where $w$ is the optimal solution and $b$ is 
the bias parameter. These parameters are derived to classify the data correctly. By accordingly, the SVM construct the constraint quadratic optimization problem that minimizes the training and generalization error by,

$$
\min _{w, \zeta} \Phi(w, \zeta)=\frac{1}{2}\|w\|^{2}+C \sum_{i=1}^{l} \zeta_{i}
$$

where $\zeta_{i}, i=1$, are non-negative slack variable and $C$ is the regularization parameter which controls the penalty incurred by each misclassified point in the training set [8], [9]. In this paper, a radial basis function (RBF) is used as the kernel function to classify the voltage sag as whether is caused by fault in power system or starting of the induction motor. The $\mathrm{RBF}$ equation is as follows,

$$
K\left(x_{i}, x_{j}\right)=\exp \left(\gamma\left\|x_{i}-x_{j}\right\|^{2}\right), \gamma>0
$$

Here, $\gamma$ is the parameter to be optimized in the classification process. The voltage sag data are divided into training and testing sets.

\section{CROSS VALIDATION AND TESTING}

Cross validation is a method that is used to select the best parameter values of $\mathrm{C}$ and $\gamma$ for the training set as shown in Eq. (4) and Eq. (5). In cross validation, for a given training set, the data set into $k$ subsets or folds of equal size of which $k-1$ subsets are used for the training and the remainder one subset is used for validation of the model. This process is repeated $k$ times until each subset have been used once for prediction.
The best parameter of $C$ and $\gamma$ used in the research are exponentially growing sequences. The range of $C$ and $\gamma$ are $C$ $=\left\{2^{-5}, 2^{-4}, \ldots, 2^{4}, 2^{5}\right\}$ and $\gamma$ is $\gamma=\left\{2^{-5}, 2^{-4}, \ldots, 2^{4}, 2^{5}\right\}$ respectively with step size of 0.1 for both exponentials.

\section{RESULTS AND DISCUSSION}

The results from of this research are presented and discussed in this section. Total of 105 and 222 data sets for both types of disturbances were generated from the IEEE 30 bus and IEEE 34 bus respectively. These data are divided into training and testing sets which are $70 \%$ of those samples are used for training and the remainder $30 \%$ for testing in the classification parts. Table I tabulate details parameters and the corresponding accuracy from the RBF based SVM classification for the IEEE 30 bus system. For each 3 -fold, 5-fold and 10-fold, the best fitting value of $C$ and $\gamma$ is presented accordingly. The classification accuracy (CV) is taken from the testing of the proposed RBF model. The accuracy for 3-fold, 5-fold and 10-fold are above $90 \%$ for the IEEE 30 bus system. On the other hand, for the IEEE 34 bus, the 10 -fold gives the best accuracy which is $95.71 \%$.

Table II tabulates for both IEEE 30 bus and IEEE 34 bus distribution system for energy as the input features. The best accuracy for IEEE 30 bus and IEEE 34 bus system is $98.57 \%$ and $94 \%$ respectively.

A comparison between ANN classification [14] and SVM performance are presented in Table III. The classification by SVM gives a higher accuracy in both features than the ANN for both systems. Therefore, it can be concluded that SVM with RBF classification is more superior as compared to ANN for voltage sag classification in this research.

TABLE I: MAXIMUM AND MinimuM VOLTAGE FEATURES

\begin{tabular}{l|lll|lll}
\hline \multirow{2}{*}{ SVM Result } & IEEE 30 Bus & \multicolumn{5}{l}{ IEEE 34 Bus } \\
\cline { 2 - 7 } & 3-fold & 5-fold & 10-fold & 3-fold & 5-fold & 10-fold \\
\hline$C$ & $2^{-1.1}=0.466$ & $2^{-1.3}=0.406$ & $2^{-0.6}=0.659$ & $2^{-3}=0.125$ & $2^{1.2}=2.297$ & $2^{-2}=0.25$ \\
\hline$\gamma$ & $2^{0.3}=1.231$ & $2^{-0.3}=0.812$ & $2^{-1}=0.5$ & $2^{-1.9}=0.267$ & $2^{1.8}=3.482$ & $2^{1.9}=3.732$ \\
\hline CV Accuracy (\%) & 94.29 & 95.71 & 95.71 & 81.08 & 95.50 & 97.30 \\
\hline \multicolumn{7}{|c}{} \\
\multicolumn{7}{|c}{ TABLE II: ENERGY FEATURE } \\
\hline \multirow{7}{*}{ SVM Result } & IEEE 30 Bus & IEEE 34 Bus \\
\cline { 2 - 7 } & 3-fold & 5 -fold & 10-fold & 3-fold & 5-fold & 10-fold \\
\hline$C$ & $2^{3.3}=9.849$ & $2^{3.5}=11.313$ & $2^{3.5}=11.313$ & $2^{4}=16$ & $2^{3.7}=12.996$ & $2^{3.6}=12.125$ \\
\hline$\gamma$ & $2^{2.5}=5.656$ & $2^{2.4}=5.278$ & $2^{2.5}=5.657$ & $2^{5}=32$ & $2^{4.6}=24.251$ & $2^{4.6}=24.251$ \\
\hline CV Accuracy (\%) & 92.87 & 98.57 & 90.00 & 91.33 & 91.33 & 94.00 \\
\hline
\end{tabular}

TABLE III: COMPARISON BETWEEN SVM AND ANN ACCURACY PERFORMANCE

\begin{tabular}{c|cc|cc}
\hline & IEEE 30 Bus & \multicolumn{2}{c}{ IEEE 34 Bus } & \\
\hline \multirow{2}{*}{ Features } & SVM & ANN & SVM & ANN \\
& Accuracy (\%) & Accuracy (\%) & Accuracy (\%) & Accuracy (\%) \\
\hline Minimum and maximum & 95.71 & 90.48 & 97.30 & 86.36 \\
\hline Energies & 98.57 & 94.29 & 94.00 & 84.09 \\
\hline
\end{tabular}

\section{CONCLUSIONS}

A new method of classification for voltage sags has been presented in this paper. The proposed classification is based on the MRA anlysis and SVM classification method. Further, to reveal the accuracy of the proposed classification, it has been evaluated with standard test simulated using IEEE 30 bus and IEEE 34 bus distribution system. The proposed classification has been found suitable in each case by using either maximum and minimum voltage or energies as the input features to predict the cause of voltage sag. In addition, the superiority of the proposed classification is illustrated by comparing it with the well known ANN classification method. For further research, this study can compare SVM with other artificial intelligence techniques like generic algorithm and particle swam optimization. 


\section{ACKNOWLEDGEMENT}

The authors would like to express heartiest appreciations to Universiti Teknologi MARA and its Research Management Institute for the support during the research. The authors also gratefully acknowledge MOSTI (E-Science 03-01-01-SF0098) for financial support on the project.

\section{REFERENCES}

[1] M. H. J. Bollen, "Voltage sags in three-phase systems," IEEE Power Engineering Review, pp. 8-15, 2001.

[2] M. H. J. Bollen, Understanding Power Quality Problems, IEEE Press, 2000.

[3] M. F. McGranaghan and D. R. Mueller, "Voltage sags in industrial systems," IEEE Trans. on Industry Applications, vol. 29, no. 2, pp. 397-403, 1993.

[4] Recommended Practice for Monitoring Electric Power Quality, IEEE Standard 1159-1995.

[5] C. Li, T. Tayjasanant, W. Xu, and X. Li, "Method for voltage sag source detection by investigating slope of the system trajectory," IEE Proceedings of Generation, Transmission and Distribution, vol. 150, no. 3, pp. 367-372, 2003.

[6] P. G. V. Axelberg, I. Y. H. Gu, and M. H. J. Bollen, "Support vector machine for classification of voltage disturbances," IEEE Trans. on Power Delivery, vol. 22, issue 3, pp. 1297-1303, 2007.

[7] P. Janik and T. Lobos, "Automated classification of power-quality disturbances using SVM and RBF networks," IEEE Trans. on Power Delivery, vol. 21, pp. 1663-1669, 2006

[8] W. Tong, X. Song, J. Lin, and Z. Zhao, "Detection and classification of power quality disturbances based on wavelet packet decomposition and support vector machines," in Proc. the $8^{\text {th }}$ Int. Conference on Signal Processing, 2006, vol. 4, pp. 16-20.

[9] W. M. Lin, C. H. Wu, C. H. Lin, and F. S. Cheng, "Classification of multiple power quality disturbances using support vector machine and one-versus-one approach," in Proc. the Int. Conference of Power system Technology, 2006, pp. 1-8.

[10] P. Thakur, A. K. Singh, and R. C. Bansal, "Novel way for classification and type detection of voltage sag," IET Generation, Transmission \& Distribution, vol. 7, no. 4, pp. 402-404, 2012.
[11] F. Xu, Y. Zhang, H. Yang, and X. Xiao, "Classification for voltage sags based on generalized s-transform," in Proc. 9th Int. Conf. on Fuzzy Systems and Knowledge Discovery, 2012, pp. 1760-1763.

[12] M. Manjula, A. V. R. S. Sarma, and S. Mishra, "Detection and classification of voltage sag causes based on empirical mode decomposition," in Proc. 2011 Annual IEEE India Conference, pp. $1-5$.

[13] V. Vapnik, Statistical Learning Theory, New York: Wiley, 1998.

[14] G. Y. Lv and X. D. Wang, "Voltage sags detection and identification based on phase-shift and RBF neural network," in Proc. Fourth Int. Conf. on Fuzzy Systems and Knowledge Discovery, 2007, pp. 684-688.

H. Ismail received her B.Eng. (Hon) degree from Universiti Teknologi MARA in 2009. She works as a aresearch assistance at the Faculty of Electrical Engineering, Universiti Teknologi MARA, Malaysia. Her project involves the prediction of voltage sag source using signal processing applications.

Z. Zakaria received the B.Eng. degree in electrical engineering from Universiti Teknologi Malaysia in 1989, then received the M.Sc. degree from Napier University, Edinburgh in 1997 and the PhD degree from University of Strathclyde, UK in 2005. She is currently an associate professor in the Centre of Power Engineering Studies, Universiti Teknologi MARA, Malaysia and also a senior member of IEEE. Her research interests include load profiling, power system analysis and AI application in power system.

N. Hamzah received her B.Eng. (Hon) degree from University of Wales Inst. of Science and Technology, UK in 1988 and the M.Sc. degree in powe system from University of Malaya, Malaysia in 1993. She obtained a PhD degree in electrical engineering (electrical and electronics) from the Universiti Kebangsaan Malaysia in 2006. She is an associate professor at the Faculty of Electrical Engineering, Universiti Teknologi MARA, Malaysia. She is actively publishing technical papers in the national and international conferences and journals and also serves as a referee in an international journal. Her research interests include power quality studies, application of advanced signal processing in power system and artificial neural network studies. 
Solar Energy 
